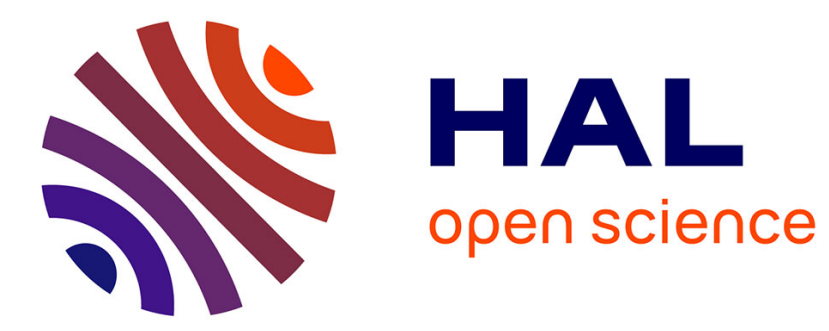

\title{
Comprendre les freins à la consommation de spectacles vivants à travers la conception individuelle de l'art.
}

\author{
Céline Gallen, Danielle Bouder-Pailler
}

\section{To cite this version:}

Céline Gallen, Danielle Bouder-Pailler. Comprendre les freins à la consommation de spectacles vivants à travers la conception individuelle de l'art.. 2010. hal-00455403

\section{HAL Id: hal-00455403 \\ https://hal.science/hal-00455403}

Preprint submitted on 10 Feb 2010

HAL is a multi-disciplinary open access archive for the deposit and dissemination of scientific research documents, whether they are published or not. The documents may come from teaching and research institutions in France or abroad, or from public or private research centers.
L'archive ouverte pluridisciplinaire HAL, est destinée au dépôt et à la diffusion de documents scientifiques de niveau recherche, publiés ou non, émanant des établissements d'enseignement et de recherche français ou étrangers, des laboratoires publics ou privés. 
EA 4272

\title{
Comprendre les freins à la consommation de spectacles vivants à travers la conception individuelle de l'art
}

\author{
Céline Gallen(*) \\ Danielle Bouder-pailler $\left(^{*}\right)$
}

$2010 / 10$

$\left({ }^{*}\right)$ LEMNA - Université de Nantes / CNAV - INED - Paris

Laboratoire d'Economie et de Management Nantes-Atlantique Université de Nantes

Chemin de la Censive du Tertre - BP 52231 44322 Nantes cedex 3 - France

www.univ-nantes.frliemn-iae/recherche 
Comprendre les freins à la consommation de spectacles vivants à travers la conception individuelle de l'art

Revue Décisions Marketing ${ }^{\circ}$ 58, 2010

\author{
Céline Gallen \\ Maître de Conférences \\ Laboratoire d'Economie et de Management de Nantes Atlantique \\ Université de Nantes \\ Institut d'Economie et de Management de Nantes - IAE \\ Chemin de la Censive du Tertre \\ BP 52231 - 44322 Nantes Cedex 3 France \\ Tél : 0240141658 \\ celine.gallen@univ-nantes.fr \\ Danielle Bouder-Pailler \\ Maître de Conférences - HDR \\ Laboratoire d'Economie et de Management de Nantes Atlantique \\ Université de Nantes \\ Chemin de la Censive du Tertre \\ BP 52231 - 44322 Nantes Cedex 3 France \\ danielle.pailler@univ-nantes.fr
}




\title{
Comprendre les freins à la consommation de spectacles vivants à travers la conception individuelle de l'art
}

\begin{abstract}
Résumé
L'objectif de notre recherche est d'explorer la conception que les individus ont de l'art afin d'identifier les freins à la consommation de spectacles vivants. Le discours de soixante-huit non-consommateurs de spectacles vivants révèle deux conceptions de la relation à l'art: l'une individuelle et affective associée à la peinture et à la musique, l'autre collective et cognitive associée aux spectacles vivants. Ces dissonances qui renvoient aux dimensions de la valeur perçue de l'expérience de consommation pourraient expliquer les freins à la consommation de spectacles vivants. Des stratégies visant à créer des contextes expérientiels sont alors proposées aux professionnels de la culture afin de favoriser l'appropriation de cette forme d'art par les non-publics.
\end{abstract}

\section{Mots clés}

Consommation culturelle, spectacles vivants, représentations mentales, dissonance cognitive, valeur perçue, marketing expérientiel.

\section{Understanding barriers to performing arts attendance through individual art conception}

\begin{abstract}
The objective of our research consists in examining how art is conceived by individuals, in order to identify barriers to performing arts attendance. The analysis of sixty-eight interviews with non-consumers of performing arts reveals two conceptions of art : one being both individual and emotional which is connected to the fine arts and music ; the other being collective and cognitive, associated with performing arts. Those cognitive dissonances impact on dimensions of perceived value of experience and could explain barriers to consumption. Strategies towards cultural industry management are then discussed in order to make appropriation easier and to improve consumers' experience.
\end{abstract}

\section{Key-words}

Cultural consumption, performing arts, mental representations, cognitive dissonance, perceived value, experiential marketing. 


\section{Introduction}

Les professionnels de la culture cherchent aujourd'hui à comprendre pourquoi le spectacle vivant est en crise ${ }^{1}$. En effet, cette forme artistique - caractérisée par la présence physique d'au moins un artiste du spectacle lors de la représentation en public d'une œuvre de l'esprit - connaît un taux de pénétration sensiblement identique depuis un demi-siècle : pour le théâtre, par exemple, il stagne à $16 \%{ }^{2}$. Face aux transformations comportementales et sociétales des pratiques, à l'offre croissante et diversifiée des formes d'expression artistique, aux mutations technologiques liées à la révolution du numérique, les professionnels peinent à diversifier et à renouveler leur public. Ces constats questionnent notamment les chercheurs en marketing et en sociologie qui tentent de proposer aux professionnels de nouvelles pistes de réflexion et d'action. Ainsi, le modèle de cohérence des comportements culturels, sous-tendu par la notion bourdieusienne d'habitus, se trouve aujourd'hui remis en cause par la notion de « dissonances culturelles » (13). En effet, si la société française a longtemps établi des oppositions symboliques entre « culture » et « sous-culture », « arts mineurs » et « arts majeurs », le rapport à la culture dite «savante » (opéra, théâtre, musique classique, danse, musée, etc.) s'est profondément modifié en l'espace de trente ans en grande partie en raison de la massification de l'enseignement secondaire (5). Aussi, même si certaines pratiques (dans le domaine musical notamment) nécessitent des compétences encore inégalement distribuées dans l'espace social, la culture savante ne relèverait plus exclusivement de pratiques majoritaires au sein des populations à fort capital scolaire et des classes dominantes, celles-ci étant sous l'influence des cultures de masse. En effet, la démocratisation et la spectacularisation de la politique culturelle impulsées sous Jack Lang dans les années quatre-vingts, la démocratisation scolaire, le recul de l'enseignement des humanités au profit des matières technico-scientifiques et la montée en puissance du pouvoir des médias ont, selon Donnat, contribué à brouiller les frontières entre culture populaire et culture cultivée (6). D'autre part, les mobilités sociales, scolaires et professionnelles, les contraintes et interactions professionnelles, soumettent les individus, tout au long de leur existence, à des influences qui vont aboutir à des pratiques culturelles hétérogènes (13). Dès lors, le marketing reconnaît que le débat ne se situe plus seulement entre les classes, mais également dans les pratiques et préférences des individus (13). Parallèlement, la sociologie des pratiques culturelles pointe la nécessité de prendre en compte les attitudes et modes d'appréhension des

\footnotetext{
${ }^{1}$ Rapport Latarjet, "Pour un débat national sur l'avenir du spectacle vivant", Ministère de la Culture et de la communication, avril 2004.

${ }^{2}$ Source : Enquête participation culturelle et sportive, Insee, mai 2003.
} 
objets des pratiques culturelles, les frontières entre les groupes sociaux ne découlant pas nécessairement des objets culturels eux-mêmes mais de la manière de se les approprier (5). En effet, l'étude des modes de perception esthétique associés à l'expérience artistique nécessite une analyse spécifique prenant en compte, plus que pour les objets de consommation courante, les sensations subjectives dans lesquelles la dimension symbolique et imaginaire, ainsi que les facteurs émotionnels et affectifs, occupent une place importante (12). L'expérience artistique décrite par Carù et Cova (3) comme une « immersion totale et foudroyante qui transforme l'individu qui la vit » nécessite, dans la réalité quotidienne, un travail d'appropriation de la part des individus.

C'est dans cette perspective que nous nous proposons d'explorer les processus psychologiques explicatifs des comportements de consommation culturelle, afin de comprendre pourquoi les non-consommateurs ne se laissent pas toujours convaincre et séduire par l'offre proposée dans le domaine des spectacles vivants.

Au regard de cet objectif, deux nécessités apparaissent. Il est tout d'abord essentiel de situer le spectacle vivant dans son ensemble évoqué, dans la mesure où l'on ne peut envisager de comprendre pourquoi les individus ne vont pas voir des spectacles sans analyser les autres formes de consommation culturelle. On ne peut en effet isoler les pratiques artistiques les unes des autres au regard des substitutions qui existent car, au sein de cet ensemble évoqué, à de mêmes motivations (recherche de sensations, par exemple), des réponses artistiques différentes peuvent être apportées. D'autre part, certaines pratiques impliquent une relation principalement individuelle (telle que la lecture) ou en cercle restreint (la contemplation d'un tableau par exemple), alors que d'autres s'inscrivent dans une relation avec l'artiste mais également avec le public (ce qui est le cas des spectacles vivants). C'est pour cette raison qu'il faut situer les différentes expériences artistiques les unes par rapport aux autres et identifier les dimensions qui interviennent dans les arbitrages que font les individus entre les diverses formes d'expériences artistiques. La conception de l'art qu'ont les non-consommateurs pourrait ainsi contribuer à expliquer le fait qu'ils n'assistent pas à des spectacles vivants.

Connaitre la nature des freins associés à la consommation culturelle pour comprendre le contenu de l'expérience nous apparaît comme la seconde nécessité. En effet, une des caractéristiques de cette consommation est qu'elle repose sur la recherche d'une expérience avec des objectifs d'ordre à la fois fonctionnel et esthétique (10). Ainsi, les produits/services culturels comportent des composantes informationnelles (caractéristiques pouvant faire l'objet d'une évaluation précise avant l'achat, telles que le nom des artistes, le lieu de représentation), mais aussi des composantes 
expérientielles (caractéristiques ne pouvant faire l'objet d'une évaluation précise que lors du vécu de l'expérience, telles que les émotions ressenties). Il nous semble donc pertinent d'analyser le contenu de l'expérience elle-même pour mieux comprendre le processus de décision (1), et en cela nous intéresser à la signification symbolique et à la vision subjective de l'interaction entre le sujet et l'activité (10). Des différences individuelles pourront ainsi être observées et expliquer un évitement de la consommation.

Dès lors, les objectifs de notre recherche se situent à deux niveaux complémentaires, à savoir :

- explorer la conception de l'art des non-consommateurs de spectacles vivants afin de situer ces derniers, dans l'ensemble spontanément évoqué, par rapport aux autres expériences artistiques ;

- identifier les freins à la consommation de spectacles vivants afin de mieux comprendre pourquoi certains consommateurs s'en détournent.

Guidés par ces objectifs, nous avons mené une étude exploratoire. L'analyse de contenu de soixante-huit entretiens non-directifs permet d'identifier deux conceptions de l'art. La mobilisation du concept de représentations d'une part montre que les freins à la consommation culturelle peuvent s'expliquer par l'existence de dissonances cognitives au sein de ces conceptions (ou représentations). Ces dissonances qui renvoient aux dimensions de la valeur perçue de l'expérience de consommation nous permettent ensuite de mieux comprendre la nature des freins à la consommation de spectacles vivants. Ces réflexions donnent lieu à des propositions issues du marketing expérientiel pour les professionnels afin de favoriser l'appropriation de cette forme d'art par les nonconsommateurs.

\section{La conception de l'art chez les non-consommateurs de spectacles vivants}

Le caractère exploratoire de l'étude nous a conduits à adopter une méthodologie qualitative (16), notre objectif étant de comprendre et non de mesurer. Une démarche inductive a donc été adoptée car elle permet, en partant d'observations limitées, de confronter les observations à la théorie. Afin d'explorer la conception de l'art chez les non-consommateurs de spectacles vivants, soixante-huit entretiens non-directifs ont été 
réalisés. L'objectif était double : (1) recueillir un discours spontané sur l'art, (2) favoriser l'expression sur le contenu de l'expérience esthétique afin de repérer les freins. Les répondants ont dès lors été sollicités pour s'exprimer sur la place de l'art dans leur vie. Les données ont ensuite été traitées par un logiciel d'analyse de données textuelles (encadré 1).

[insérer encadré 1]

\section{Encadré 1 : Méthodologie de traitement des données}

Le traitement des données textuelles a été effectué selon la méthodologie ALCESTE (Analyse des Lexèmes Co-occurrents dans les Enoncés Simples d'un Texte), spécifiquement adaptée pour l'analyse d'un corpus de texte homogène (ici des entretiens non-directifs). Elle permet d'analyser la distribution du vocabulaire obtenue à partir des entretiens et de réaliser des analyses lexicographiques (comptages sur des fréquences d'apparition de mots) et lexicales (recherche de significations par la contextualisation des mots).

Dans un premier temps, le corpus est découpé en "unités de contexte élémentaires" (u.c.e.) reflétant une idée centrale, de manière à repérer les thèmes et la structure du discours. Le logiciel procède ensuite au moyen d'une classification descendante hiérarchique à la partition du corpus en classes illustrées par des formes représentatives (mots pleins, mots-outils, mots étoilés, verbatims). Cette partition fait ensuite l'objet d'une représentation graphique qui met en avant l'existence d'associations reflétées par les énonciations des répondants.

Les résultats de l'analyse des données par ALCESTE ont été interprétés par deux chercheurs, de manière indépendante.

Les soixante-huit sujets (trente-quatre hommes et trente-quatre femmes) retenus pour l'étude sont des non-consommateurs relatifs de spectacles vivants relevant de pratiques dites cultivées (opéra, théâtre, musique classique...). En effet, c'est dans ces pratiques que les freins sont les plus importants et que les professionnels ressentent la nécessité de renouveler leurs publics (généralement vieillissants). Ainsi, les non consommateurs ont été sélectionnés selon deux critères : (1) une variable comportementale : la fréquence de consommation (ici, la non consommation depuis cinq ans de spectacles vivants dans le cadre de pratiques dites cultivées), (2) une variable psychologique : la sensibilité à l'art. Celle-ci fut appréhendée par un discours spontané des personnes sur leur proximité affective et leurs connaissances cognitives liées à l'art, qu'elles soient le fait d'une transmission familiale ou d'une éducation extérieure. Enfin, en vertu du caractère exploratoire inductif de notre recherche, nous avons veillé à diversifier les profils sociodémographiques des interviewés (annexe 1). Cependant, le postulat d'une hétérogénéité des itinéraires et des positions sociales à l'intérieur d'un même groupe social (6) - liée aux profondes mutations sociales des cinquante dernières années (progrès de la scolarisation, développement du chômage, précarisation de l'emploi, diversification des situations familiales...) - nous conduit à privilégier l'étude des 
préférences et pratiques individuelles.

\section{L’identification de deux dimensions de la conception de l'art}

L'analyse statistique des données textuelles sous ALCESTE permet d'identifier quatre classes lexicales. Le dendogramme des classes stables obtenu par classification descendante hiérarchique montre que les classes 1, 2 et 4 sont liées, alors que la classe 3 est plus indépendante (annexe 2).

Il ressort des analyses deux conceptions de l'art : d'une part, une conception individuelle et affective de l'art de laquelle le spectacle vivant est absent et, d'autre part, une conception collective et cognitive de l'art à laquelle le spectacle vivant est associé (tableau 1).

\section{- Une conception individuelle et affective de l'art}

La classe 1 évoque les arts plastiques. C'est d'abord l'objet esthétique qui est désigné ("tableau" : 92 occurrences, "peinture" : 66, "photo" : 27, "toile" : 26, "œuvre" : 18, "sculpture" : 15, "gravure" : 14). Les attributs évoqués sont le style ("abstrait", "impressionnisme", "figuratif") et la composition ("couleur", "noir", "pastel", "aquarelle"...). La création est personnifiée à travers le "peintre" (39 occurrences), parfois son nom est précisé ("Picasso", "Van Gogh"). Le vocabulaire est exclusivement positif et se centre sur ce qui lie les deux composantes évoquées (l'œuvre et le créateur) à l'individu interviewé. Les évocations sont affectives: "aimer" (79 occurrences), "plaire" (23), "beau" (20). Le premier mot-outil de la classe est l'adjectif possessif "mon" et les trois segments répétés les plus fréquents sont: "j’ai", "j’aime", "je suis". Cela semble témoigner d'une représentation de l'individu comme étant isolé dans sa relation esthétique à l'objet (l'œuvre), désiré pour lui-même.

On retrouve dans la classe 2 la relation individuelle à l'objet artistique. Comme dans le premier contexte, c'est la relation émotionnelle entre l'œuvre et l'individu qui fait l'objet de l'essentiel du discours, mais la classe est cette fois clairement centrée sur la musique. L'œuvre musicale est évoquée par des styles très différents : "musique classique", "jazz", "techno", "contemporaine"... L'objet artistique est là encore perçu pour lui-même mais, contrairement à la classe 1, non associé à l'artiste : le mot "musicien" est cité seulement 8 fois. Les mots les plus cités, par ordre décroissant d'occurrence, caractérisent les bénéfices perçus vis-à-vis de l'œuvre : "procurer", "émotion", "plaisir", "bonheur", "découverte", "magnifique", "beauté", "triste", "détendre", "pleurer"... Aucune u.c.e. ne fait le lien avec une représentation collective 
de la musique : le mot "concert" n'est pas cité dans cette classe. Cette dernière traduit une relation fusionnelle entre l'œuvre musicale et l'interviewé. Les deux principaux mots-outils de cette classe confortent cette analyse : "dans" et "suis".

On notera en outre que l'on retrouve dans les deux premières classes, l'illustration de la complexité du statut du produit/service culturel (1). Il est évoqué à la fois à travers un regard analytique, fondé sur une somme d'attributs (styles, compositions...) (classe 1), mais aussi à travers une appréhension holiste de l'expérience, évaluée par les bénéfices émotionnels (classe 2).

\section{- Une conception collective et cognitive de l'art}

La classe 4 correspond à une conception collective de l' "art" (u.c.e. citée 395 fois) au sein de laquelle l'"artiste" trouve une place importante (232 occurrences). De nombreuses u.c.e. sont liées à la composante sociale de la consommation culturelle : "gens", "monde", "société", "personne", "regard"... Les attentes exprimées sont ici d'ordre cognitif: "comprendre", "reconnaître", "permettre", "servir", "intéressant", "savoir", "besoin", "règle"... L'individu interviewé ne s'implique pas dans ces évocations. Il semble en être absent : il n'y a pas d'évocation personnelle. Les motsoutils spécifiques de cette classe sont par ordre décroissant: "pouvoir", "falloir", "devoir". Il y aurait une forme de mise à distance entre l'individu qui s'exprime et l'art désigné par "les autres".

Enfin, la classe 3, plus indépendante des autres classes, est centrée sur le "spectacle" (142 occurrences) et toutes les formes qu'il peut avoir : "théâtre", "concert", "opéra"... Il évoque aussi d'autres pratiques culturelles: "cinéma", "exposition", "musée". L'ensemble des formes de consommation collective semble amalgamé. C'est une évaluation cognitive qui prévaut : le verbe le plus cité est "intéresser". Le contexte de la consommation est très présent dans les évocations de cette classe: le lieu (42 occurrences), le moment (le soir, 26 occurrences), le fait d'être seul (31) ou accompagné par des amis (30 occurrences).

[insérer tableau 1] 


\section{Tableau 1}

Résumé des analyses des données de la conception de l'art chez les nonconsommateurs de spectacle vivant.

\begin{tabular}{|c|c|c|c|c|c|}
\hline Classe & $\begin{array}{c}\% \\
\text { u.c.e. }\end{array}$ & $\begin{array}{c}\text { Principales formes } \\
\text { représentatives de la } \\
\text { classe } \\
\text { (par ordre décroissant du } \\
\text { nombre d'occurrences) }\end{array}$ & $\begin{array}{c}\text { Contenu des } \\
\text { évocations }\end{array}$ & $\begin{array}{c}\text { Evocation } \\
\text { spontanée } \\
\text { du spectacle } \\
\text { vivant }\end{array}$ & $\begin{array}{l}\text { Sens commun partagé } \\
\text { entre les classes }\end{array}$ \\
\hline 1 & $16 \%$ & $\begin{array}{l}\text { tableau } \\
\text { aimer } \\
\text { peinture } \\
\text { peintre } \\
\text { œuvre } \\
\text { couleur } \\
\text { sculpture } \\
\text { maison }\end{array}$ & $\begin{array}{c}\text { Relation affective } \\
\text { « individu } / \\
\text { « œuvre plastique » }\end{array}$ & non & \multirow{2}{*}{$\begin{array}{c}\text { Conception } \\
\text { individuelle et } \\
\text { affective de la relation } \\
\text { esthétique } \\
\text { (dimensions } \\
\text { intrinsèques) } \\
\text { sans évocation de } \\
\text { l'artiste : relation } \\
\text { directe, sensorielle et } \\
\text { fusionnelle avec } \\
\text { l'œuvre }\end{array}$} \\
\hline 2 & $6 \%$ & $\begin{array}{l}\text { musique } \\
\text { écouter } \\
\text { vivre } \\
\text { jour } \\
\text { procurer }\end{array}$ & $\begin{array}{c}\text { Lien émotionnel } \\
\text { « individu »/ } \\
\text { « œuvre musicale » }\end{array}$ & non & \\
\hline 4 & $41 \%$ & $\begin{array}{c}\text { art } \\
\text { artiste } \\
\text { gens } \\
\text { comprendre } \\
\text { ouvre } \\
\text { faire } \\
\text { chose }\end{array}$ & $\begin{array}{l}\text { Relation cognitive } \\
\text { avec l'artiste : mise } \\
\text { à distance entre } \\
\text { l'individu qui } \\
\text { s'exprime et l'art } \\
\text { désigné par « les } \\
\text { autres » (univers du } \\
\text { spectacle) }\end{array}$ & oui & \multirow{2}{*}{$\begin{array}{c}\text { Conception collective } \\
\text { et cognitive } \\
\text { (dimensions } \\
\text { extrinsèques) } \\
\text { avec évocation des } \\
\text { artistes et des } \\
\text { « autres ». } \\
\text { Absence de l'individu } \\
\text { (observateur) }\end{array}$} \\
\hline 3 & $36 \%$ & $\begin{array}{l}\text { spectacle } \\
\text { intéresser } \\
\text { aller } \\
\text { voir } \\
\text { théâtre } \\
\text { concert } \\
\text { lieu }\end{array}$ & $\begin{array}{c}\text { Le spectacle } \\
\text { considéré comme } \\
\text { une activité sociale }\end{array}$ & oui & \\
\hline
\end{tabular}

Ces analyses permettent d'identifier les dimensions qui structurent la conception que les individus ont de l'art, et d'y situer les spectacles vivants (premier objectif de cette recherche). Nous allons maintenant interpréter ces «conceptions » à la lumière des concepts de représentations et de valeur perçue de l'expérience afin d'identifier les freins à la consommation de spectacles vivants (second objectif).

\section{L'identification des freins à la consommation de spectacles vivants}

L'interprétation par l'individu d'une proposition culturelle telle que les spectacles 
vivants mobilise deux types de ressources: (1) des ressources individuelles d'ordre physiologique relevant de la perception sensorielle à travers les cinq sens, et (2) des ressources individuelles d'ordre cognitif qui vont déclencher des processus à la fois affectifs et cognitifs (12). Ces dernières sont mobilisées grâce aux représentations mentales qui permettent l'interprétation des stimuli dans l'environnement par comparaison de leurs caractéristiques avec les représentations stockées en mémoire (9). Parallèlement, la désignation affective de la représentation est activée, ce qui a pour effet d'influencer la valeur perçue de l'expérience (11). Les représentations s'accompagnent alors d'attitudes qui, par le biais de la valeur perçue, s'exprimeront dans des comportements (figure 1). Or, l'analyse des résultats tend à montrer qu'au sein des représentations de l'art, il existe des dissonances cognitives reposant sur des divergences de conception de la relation à l'art. Ces dissonances qui renvoient aux dimensions de la valeur perçue de l'expérience de consommation pourraient expliquer les freins à la consommation de spectacle vivant.

[insérer figure 1]

\section{Figure 1}

Proposition de chaîne causale pour expliquer les freins à la consommation de spectacles vivants

Perception
des stimuli
associés à
l'œuvre $\quad \begin{gathered}\text { Activation des } \\ \text { représentations } \\ \text { mentales } \\ \text { Dissonances } \\ \text { cognitives? }\end{gathered} \quad \begin{gathered}\text { Valeur } \\ \text { perçue de } \\ \text { l'expérience }\end{gathered} \quad \begin{gathered}\text { Comportement } \\ \text { Engagement / } \\ \text { évitement }\end{gathered}$

\section{Les représentations associées au spectacle vivant}

La sollicitation des ressources individuelles, cognitives et affectives, pour percevoir l'objet apparait nettement dans l'analyse des discours des répondants à travers les formes représentatives des classes (tableau 1). Elle constitue le "style esthétique", à savoir la manière personnelle de percevoir la dimension esthétique face à un stimulus culturel (12). Le consommateur identifie ainsi l'objet comme étant esthétique ou non, en concordance avec ses représentations mentales (encadré 2). Les dimensions individuelles et collectives des représentations qui apparaissent dans les discours des répondants montrent notamment qu'ils contextualisent leur consommation en $\mathrm{y}$ associant des faits objectifs (situations, personnes, actions) issus d'un ensemble de normes ou d'attentes appartenant à l'univers dans lequel se situe l'expérience. Les 
individus vont chercher à donner un sens à la consommation en attribuant d'abord une signification aux objets (peinture, œuvre musicale) et aux actions (fait d'aller au théâtre, dans une salle de spectacle), puis en les interprétant dans leur univers social. L'analyse des résultats nous a ainsi permis d'identifier des représentations différentes chez l'individu dans sa relation à l'art.

[insérer encadré 2]

\section{Encadré 2 : Définition des représentations mentales}

Les représentations mentales individuelles, issues de la psychologie cognitive, sont définies en marketing comme un produit cognitif et un processus mental individuel, issus de l'interaction de l'individu avec son environnement (9). Elles permettent de coder la signification des stimuli qui en émanent et de conserver cette information en mémoire. Elles se distinguent: des croyances et des schémas de référence (qui ne recouvrent que l'aspect structurel de la cognition); de la perception (qui produit un processus dont les représentations constituent le résultat); des images mentales (qui sont considérées comme une forme particulière de représentations). Organisées par catégories, elles servent de filtre à la perception et de référence à l'interprétation.

Ainsi, les classes 1 et 2 (tableau 1) sont centrées sur l'individu dans sa relation affective et fusionnelle avec l'œuvre et montrent bien l'existence d'une sensibilité artistique, au moins à deux formes : les arts plastiques et la musique. Cependant, cette sensibilité n'est pas transposée au niveau collectif. Il ne semble pas exister de lien entre une sensibilité individuelle clairement exprimée et la consommation collective normée. Il n'y a donc pas appropriation des normes par l'individu, ce qui peut constituer un frein à sa rencontre avec l'œuvre dans un contexte collectif.

Les classes 3 et 4 (tableau 1) dans lesquelles le spectacle vivant est évoqué spontanément témoignent quant à elles d'une mise à distance entre l'individu (absent du discours) et l'objet esthétique. Dans la classe 4, l'œuvre est considérée comme un objet pour les "autres" et les normes sociales sont évoquées comme expliquant cette mise à distance. Ces évocations sont d'autant plus importantes que cette classe est celle qui recueille le plus de verbatims. Dans la classe 3 (tableau 1), le spectacle est évoqué en tant qu'objet de diffusion collective et dans sa composante situationnelle (lieu, moment, personnes).

L'analyse des discours révèle ainsi des divergences entre la conception d'un "objet" culturel et la conception des pratiques sociales relatives à celui-ci. En effet, il y a une absence de concordance entre les représentations individuelles d'un stimulus artistique et les représentations des "normes" sociales associées à ce stimulus. Cela signifie qu'il existe un écart entre la conception de 1'"objet" culturel et la conception des pratiques 
sociales relatives à celui-ci. Dans la mesure où il s'agit de relations incohérentes entre deux éléments de cognition - connaissance, idées, opinions, attitudes, croyances - sur un même objet, on peut parler de "dissonance cognitive" (8). Ainsi, une dissonance entre les représentations d'un "objet" culturel et les représentations des pratiques sociales relatives à cet objet pourrait générer des intentions de comportements défavorables et par voie de conséquence, expliquer les freins à la consommation culturelle. Nous allons montrer comment ces représentations, à travers la valeur perçue de l'expérience de consommation artistique, pourraient expliquer la non-consommation de spectacles vivants.

\section{La valeur perçue de l'expérience de consommation artistique}

Le concept de valeur perçue (encadré 3) nous a semblé pertinent pour comprendre la nature des freins à la consommation de spectacles vivants issus des représentations des différentes formes artistiques. En tant que préférence relative comparative, personnelle et situationnelle qui caractérise l'expérience de consommation d'un sujet en interaction avec un objet, la valeur perçue permet de situer les formes artistiques les unes par rapport aux autres tout en tenant compte de leur contexte de consommation.

[insérer encadré 3]

\section{Encadré 3 : Définition de la valeur perçue}

Apparu dans les recherches en marketing avec les travaux d'Holbrook (11) et désormais très connu en marketing de la culture, le concept de valeur perçue permet une approche holiste de l'expérience de consommation s'appuyant sur les perceptions des consommateurs (14).

Définie comme une préférence relative comparative, personnelle et situationnelle, résultant d'un jugement et servant à comparer des objets entre eux, la valeur perçue caractérise l'expérience de consommation d'un sujet en interaction avec un objet (11). Elle s'inscrit alors dans une approche expérientielle dans la mesure où la valeur résulte de l'expérience, mais ne la précède pas (14). Trois dimensions de la valeur perçue ont été identifiées (11). Ainsi, la valeur perçue peut :

- être intrinsèque (l'expérience de consommation est appréciée en tant que telle) ou extrinsèque (le produit est un moyen pour atteindre des fins qui lui sont extérieures);

- être orientée vers soi (l'expérience de consommation est destinée à soi, à son propre plaisir) ou vers les autres [1'expérience de consommation induit des effets sur la société (famille, collègues, amis, culture, divinité)];

- résulter d'une réponse active du sujet (manipulation active, physique ou mentale, de l'objet) ou passive (le consommateur est réactif).

Or, l'analyse du discours des représentations de l'art montre que la dissonance entre représentations individuelles et affectives de l'art d'une part et représentations collectives et cognitives d'autre part, pourrait conditionner la valeur perçue de 
l'expérience. En effet, les différentes formes d'art sont situées les unes par rapport aux autres dans un processus de pensée cohérent: la musique et les arts plastiques sont envisagés dans une relation fusionnelle et directe à l'objet esthétique alors que les spectacles vivants sont évoqués en tant qu'objet de diffusion collective. En outre, les différentes formes d'art sont discriminées selon la situation puisqu'elles s'inscrivent selon leur forme dans une pratique individuelle ou une consommation collective.

D'autre part, il apparaît dans l'analyse lexicale que le contenu discursif des répondants se structure spontanément autour des dimensions de la valeur perçue (figure 2). Ainsi, les classes 1 et 2 relatives à la peinture et à la musique (tableau 1) mettent à jour une conception de l'art :

- intrinsèque: le discours est centré sur les préférences pour les relations individuelles et isolées avec l'œuvre désirée pour elle-même ;

- orientée vers soi : la relation évoquée par l'individu avec l'œuvre est fusionnelle, directe et procure du plaisir ;

- active : la relation avec l'œuvre repose sur une implication émotionnelle forte par laquelle passe l'appropriation de l'œuvre.

Les classes 3 et 4 relatives au spectacle vivant (tableau 1) reflètent une conception :

- extrinsèque : l'œuvre est évoquée comme un objet pour "les autres" et à travers des normes collectives (lieu, moment, personnes) qui ne permettent pas une appropriation individuelle et intime.

- orientée vers les autres : la relation avec l'œuvre est distanciée, les normes sociales faisant écran à la relation; l'œuvre est considérée comme un objet pour les autres ;

- passive : l'œuvre est associée à une consommation collective dont les normes ne permettent pas une appropriation de l'œuvre par l'individu qui reste observateur.

Les représentations de l'art renvoient ainsi à l'expérience perçue de la consommation artistique. Ainsi, la dissonance entre les représentations des œuvres artistiques semble renvoyer à la dichotomie "espace privé / espace collectif" (14) qui, selon le contexte de l'expérience, modifie les interactions entre l'individu et l'objet, et donc la valeur perçue. Les différentes formes d'art sont positionnées entre elles, avec une préférence pour les relations individuelles avec l'œuvre (face à face, implication émotionnelle). Ce constat semble signifier une forme d'antinomie entre le statut perçu de l'œuvre (objet de liberté, incarnant des ruptures esthétiques, des explorations neuves dans la créativité) et le mode de transmission collectif, très normé. Ainsi, les freins à la consommation de spectacles vivants semblent être de plusieurs natures : des freins psychologiques liés à la relation 
distanciée avec l'œuvre et ses composantes situationnelles (lieu, moment, personnes) et des freins sociaux liés aux normes partagées par le groupe. Ceci peut en partie s'expliquer par le fait que, parmi les consommations culturelles, les spectacles vivants sont les plus contraints : la part du consommateur y est la plus faible en termes de choix du lieu, du moment et de la durée... à la différence du disque, du livre ou de l'œuvre d'art possédée, avec lesquels on peut entrer en contact quand on le souhaite, au moment désiré et suivant une durée choisie.

Pour certains individus, ces dissonances peuvent exister sans provoquer de gêne (c'est le cas par exemple si les sujets sont faiblement impliqués dans la catégorie de produit ou si le sujet a un seuil élevé d'acceptation de la dissonance). Ces individus surmonteront alors, voire ignoreront, la dissonance. Pour d'autres, le décalage provoquera un inconfort mental. A partir du moment où la dissonance est perçue, le sujet pourra tenter d'y remédier en vertu du principe général de refus de la discordance. Au plan psychologique, cela se traduit par un besoin de cohérence chez l'individu qui pourra adopter trois stratégies : soit il modifiera les caractéristiques de l'objet dans la représentation (en faisant du spectacle collectif un objet de proximité qu'il s'approprie, en créant un lien affectif avec la structure de diffusion, par exemple), soit il ignorera ou minimisera l'information reçue, soit il adoptera un comportement d'évitement (la nonconsommation). [insérer figure 2]

\section{Figure 2}

\section{Interprétation de l'analyse lexicale des conceptions de l'art}

Classes 1 et 2

Centrées sur la peinture et la musique

\section{Représentations individuelles et} affectives de l'art

\section{Valeur perçue de l'expérience}

Intrinsèque :

préférence pour les relations individuelles avec l'œuvre désirée pour elle-même

\section{Orientée vers soi :}

relation fusionnelle et directe entre l'individu et l'œuvre qui procure du plaisir

\section{Réponse active :}

relation avec l'œuvre reposant sur une implication émotionnelle forte par laquelle le sujet s'approprie l'œuvre.
Classes 3 et 4 Centrées sur le spectacle vivant

Représentations collectives et cognitives de l'art

Valeur perçue de l'expérience

Extrinsèque :

les spectacles vivants en tant qu'objets de diffusion collective.

Orientée vers les autres : relation distanciée avec l'œuvre, écran $=$ les normes sociales

Réponse passive dans la consommation considérée comme collective, ne permettant pas une appropriation. 


\section{Recommandations managériales pour les professionnels de la culture}

Ces résultats présentent à plusieurs égards un intérêt pour les professionnels de la culture, en particulier ceux agissant au sein d'organisations de diffusion de spectacles vivants (directeurs de structure, administrateurs, chargés des relations avec les publics, responsables de la médiation, chargés de communication, et autres managers).

L'enjeu principal qui apparaît est de favoriser une meilleure concordance entre les représentations individuelles d'un stimulus artistique et les représentations des pratiques et normes sociales associées à ce stimulus. Nous avons en effet observé que c'est bien la mise en scène sociale du spectacle vivant qui provoque des réticences de la part des spectateurs potentiels. L'existence de la dissonance qui repose sur la confrontation des représentations individuelles de cet objet culturel et de ses pratiques collectives montre que la consommation collective est perçue comme trop contrainte. Les perceptions concernant l'association du genre artistique à des lieux de diffusion (les opéras joués dans des opéras par exemple) et l'homogénéité sociale des publics (en termes d'appartenance à une classe sociale par exemple) s'opposeraient ainsi aux nouveaux modes d'appropriation individuelle de la culture.

Si ces résultats confirment ceux de Donnat et Totila en sociologie de l'art (7), la classification obtenue dans le discours des répondants permet de proposer aux professionnels des actions pour favoriser l'appropriation de l'œuvre tout en suscitant un désir de consommation collective. Carù et Cova $(3,4)$ ont à ce titre montré qu'un moyen de réduire la distance entre le consommateur et la situation de consommation culturelle consistait à faciliter l'immersion. Créer des «contextes expérientiels » avec les non-publics par de nouveaux modes de mise en relation avec l'œuvre pourrait constituer une piste d'actions visant une meilleure proximité. En nous inspirant de leurs travaux, trois types de stratégies peuvent être proposés pour renforcer la valeur perçue d'une expérience de spectacle vivant : l'action collective pour la dimension intrinsèque / extrinsèque, l'accompagnement pour la dimension orientée vers soi / orientée vers les autres et l'auto-détermination pour la dimension active / passive. Ces stratégies sont illustrées par des exemples dont pourraient s'inspirer d'autres institutions culturelles. Nous verrons que celui de «La folle journée » à Nantes est particulièrement intéressant car il illustre les trois types de stratégie. Outre ces stratégies permettant d'agir sur le contenu de l'expérience vécue, nous proposons des modalités de diffusion des œuvres, 
en amont, sous la forme de communication expérientielle afin de favoriser la rencontre avec les non-consommateurs.

\section{L'action collective}

Nos résultats confirment les tendances de nouvelles formes d'appropriation de l'art et montrent les résistances attachées aux représentations des formes d'art collectives. Autrement dit, ils illustrent la consommation paradoxale en jeu dans l'univers culturel : des pratiques de plus en plus individualisées (la formation de soi par soi, le loisir « cocooning », le développement personnel), comme une sorte de retranchement du monde dans cette quête de l'esthétique et, dans le même temps, une volonté de partage d'émotions collectives (14). Or, nous observons dans le cadre de notre recherche, que si ce désir est évoqué, la puissance de la représentation négative l'emporte sur ce souhait. Une des solutions pour les professionnels résiderait alors dans ce que Carù et Cova (4) nomme «l'action collective ».

L'action collective consiste à maintenir et développer des liens communautaires. Les contextes expérientiels doivent alors être pensés comme des lieux de lien et des lieux de rencontre permettant l'apprentissage mutuel (entre experts et novices) et le partage d'émotions (4). Ainsi, la mairie de Nantes met par exemple en place un nouveau mode de création collective intitulé "Art en partage» proposant aux populations de contribuer au processus de création d'une œuvre avec des artistes acteurs, plasticiens, photographes ou encore couturiers. A Marseille, l'initiative du $\mathrm{MuCEM}^{3}$ en 2003-2004 avec l'exposition intitulée "Parlez-moi d'Alger» a également permis de créer un lien communautaire et un partage d'émotions en attirant les populations marginalisées des quartiers Nord autour d'un thème commun.

L'action collective implique également la prise en compte des rituels associés au contexte de consommation culturelle. Ces règles de vie ont en effet une fonction de lien social pour ceux qui les partagent, mais aussi d'exclusion pour ceux qui ne les comprennent pas. Nos résultats montrent en effet que les pratiques ne sont pas perçues comme suffisamment libres puisqu'un frein à la rencontre des individus avec l'œuvre semble lié à la difficulté d'appropriation des normes sociales associées au contexte de consommation. Aussi le bouleversement ou la suppression de certains rituels pourraient

\footnotetext{
${ }^{3}$ Musée des Civilisations de l'Europe et de la Méditerranée.
} 
permettre aux novices de se sentir moins distanciés de la situation de consommation et de s'immerger plus facilement. "La folle journée », devenue un événement annuel à Nantes depuis 2002 bouleverse ainsi les rituels du traditionnel concert de musique classique avec entracte permettant de faire tomber les freins : le lieu, la cité des congrès, est un lieu non sacralisé ; les concerts sont de courte durée (45 minutes) et la possibilité de composer son propre itinéraire musical autorise une appropriation des œuvres. Par ailleurs, la relation au temps constitue un autre rituel pouvant être mobilisé pour réinventer la relation avec les publics dans la mesure où elle est intimement liée à la perception du spectacle vivant. Ainsi, lors d'une manifestation de «Lille 2000 », les spectateurs n'ont pas été conviés à une heure fixe pour le début du spectacle : on leur a proposé d'attendre collectivement le crépuscule avant que le spectacle ne débute.

Ces actions autour du lien communautaire et des rituels, si elles sont appliquées au contexte du spectacle vivant, pourraient renforcer la valorisation de l'expérience pour sa dimension intrinsèque. En effet, en favorisant le lien ou en bouleversant les rituels, l'œuvre et son contexte ne sont plus considérés comme un objet «pour les autres » mais s'en trouvent appropriés de manière plus intime.

\section{L'accompagnement}

L'accompagnement renvoie notamment à la mobilisation de référents issus d'autres contextes et servant de point d'ancrage aux consommateurs novices (4). Cette stratégie touche à la dimension orientation vers soi / orientation vers les autres de la valeur perçue de l'expérience et vise à favoriser une relation plus directe, moins distancée avec l'œuvre. Les référents permettent en effet aux individus de faire fonctionner leur imagination, de faciliter leurs propres associations et d'attribuer un sens subjectif à l'expérience selon l'histoire de chacun. Ils les aident également dans leur parcours de découverte et dans l'acquisition de compétences.

Ce travail d'accompagnement peut se faire en privilégiant une cible : par exemple les adolescents, rarement directement interpellés dans les propositions artistiques. Ainsi, un lieu de création dédié à la marionnette, le Théâtre Jeune Public (TJP) à Strasbourg se donne pour mission de renouveler des écritures scéniques tout en inventant une multiplicité de formes. Un travail d'accompagnement au théâtre est mis en œuvre pour développer et multiplier les liens avec le public adolescent par des ateliers, lectures, cafés-philo, animations, parcours de découverte du théâtre.

L'exemple de l'exposition «Hip-Hop, art de rue, art de scène » présentée en 2005 au 
MuCEM de Marseille illustre également la mobilisation de référents par le croisement des genres et l'offre de modalités d'appropriation de la proposition artistique. Au-delà d'une relecture de l'histoire du Hip-Hop (à travers des référents tels que des textes, des films, des objets, des affiches, des disques et des vêtements) proposée par l'exposition, un espace interactif multimédia avait été installé. Occupant un quart de la surface totale d'exposition, il permettait à chaque visiteur de composer sa propre musique grâce à des platines de mixage et des tapis tactiles posés au sol détectant les mouvements de pieds des visiteurs. Des capteurs permettaient également d'agir sur l'ambiance lumineuse de l'espace. Et enfin, un concours de danse permettait aux visiteurs, via des manettes de jeu vidéo, de choisir leur danseur virtuel en 3D, de le nommer, de le personnaliser et de lui faire enchainer des figures de danse. Selon Catherine Homo-Lechner, chercheur en anthropologie au MuCEM, l'exposition fut un réel succès : si elle a fait découvrir cet art de rue aux habitués des expositions, elle a aussi attiré les jeunes sensibilisés au Hip-Hop qui venaient «vivre une expérience ». Pour la plupart de ces jeunes, elle fut l'occasion d'une première prise de contact avec le musée et d'une désacralisation du lieu grâce aux modes d'appropriation qu'offrait l'espace interactif.

Enfin, «La folle journée » de Nantes offre la possibilité à des publics jeunes et novices de créer une proximité cognitive à travers le genre musical de référence (le rock, le rap, le slam...). La musique classique est ainsi revisitée dans le cadre d'ateliers de transcription : la Truite de Schubert est slamée, les concertos brandebourgeois de Bach sont interprétés avec des rythmes et accents musicaux issus du rap.

\section{L'autodétermination}

L'auto-détermination renvoie aux actions de formation des non-spectateurs et aux actions d'autonomisation par leur participation à la construction de leur expérience. Ces actions, qui s'appuient sur une participation active de l'individu dans l'expérience, permettraient de réduire la dissonance entre une conception active de l'art et une conception passive du spectacle vivant afin d'accroître la valeur perçue de l'expérience.

Un premier type d'actions consisterait à décomplexer les individus les plus novices par des actions pédagogiques de formation visant à favoriser l'appropriation cognitive des œuvres artistiques. Des initiatives existent et pourraient servir d'exemples à d'autres institutions culturelles. L'Auditorium de Milano propose ainsi un cycle de concerts nommés « Discovery » organisés en deux parties (3). Dans un premier temps, le chef d'orchestre offre au public la possibilité de découvrir une œuvre en en faisant jouer des 
extraits. Il la replace dans son contexte historique, biographique et musical. Dans un second temps, l'œuvre est interprétée dans sa globalité. "La folle journée » de Nantes joue également un rôle dans la formation des spectateurs grâce à des analyses de musicologues, des films et conférences sur les compositeurs. Ces propositions s'adressent plus spécifiquement aux non-publics qui, présents à $23 \%$ en 2008, sont venus pour la première fois découvrir de la musique classique en «live » ${ }^{4}$. En amont des concerts, la diffusion d'extraits dans la grande halle de la cité des congrès permet par ailleurs de les préparer à l'approche d'une œuvre et constitue un enjeu de « teasing » expérientiel. En outre, l'accueil des jeunes publics (scolaires) est aussi une des actions de médiation culturelle qui favorise la formation des publics et favorise la prescription au sein de la famille (accompagnement pour favoriser une démarche autonome vers la culture).

Réunir dans un même lieu la diffusion et l'enseignement pourrait constituer un autre moyen de former les non-publics. Citons le cas de "Pôle Sud» à Strasbourg (scène conventionnée pour la danse et la musique) qui développe un projet au sein duquel les axes artistiques et pédagogiques sont étroitement liés. Il s'appuie sur un programme de diffusion, de création, de formation, d'actions culturelles et de sensibilisation des publics. Une salle de spectacles de 320 places ainsi qu'un studio de danse accueillent toute l'année des concerts de jazz, des spectacles de danse contemporaine, des répétitions publiques, des conférences et des résidences d'artistes. Un nouvel équipement a permis au projet pédagogique de se renforcer grâce à deux nouveaux grands studios de danse et à cinq studios de musique destinés à l'enseignement des enfants, des adolescents et des adultes.

Ces actions de formation, au-delà de leur inscription dans un processus d'apprentissage cognitif ont également un impact symbolique. En ayant un comportement « empathique » vis-à-vis des (non-)publics, les organisations culturelles donnent des preuves de leur volonté d'ouverture et participent à la désacralisation des offres.

L'autonomisation des publics constituent un second type d'actions de la stratégie d'auto-détermination (4). Elle répond au besoin de l'individu de pouvoir interférer dans la définition des règles appliquées, d'être acteur des modalités de rencontre avec l'œuvre. Les succès des «offs » des festivals et des arts de la rue attestent de cette volonté. Ces «revendications» se trouvent aujourd'hui renforcées par le fort développement de pratiques «marginales » qui témoigne de ce souhait de liberté des

\footnotetext{
${ }^{4}$ Etude interne au Centre de Réalisations et d'Etudes Artistiques qui assure la programmation de la Folle Journée de Nantes.
} 
individus. A titre d'exemple, l'école des Beaux-Arts de Nantes organise chaque année «Les belles chaises », une exposition populaire qui valorise l'art amateur. Le principe consiste à présenter son œuvre sur une chaise, en guise de chevalet. Les (non-) publics aspirent ainsi à une consommation culturelle active : ils veulent être les initiateurs des interactions entre l'œuvre et eux-mêmes donnant ainsi de l'intensité à l'expérience (15), et cela dans un jeu de mise en questionnement des limites liées au genre. Face à ces évolutions, les organisations culturelles doivent donc inventer de nouveaux modes de mise en relation active de l'œuvre et des publics pour faire tomber les freins et créer de la proximité affective, l'enjeu étant celui de la co-création. Certains chercheurs et professionnels remettent même en cause l'existence de la notion de publics (en termes d'opposition artistes/publics) car ils ressentent la nécessité de les associer au processus de production de l'œuvre, de les rendre acteurs (2).

\section{La communication expérientielle}

Si la création de contextes expérientiels avec les non-publics constitue une piste intéressante dans la mise en place de nouveaux modes de relation avec l'œuvre, il faudrait imaginer, en amont, des modalités de diffusion des œuvres qui tendraient à favoriser la rencontre avec les non-consommateurs sous la forme de communication expérientielle.

Les artistes eux-mêmes peuvent ainsi constituer un vecteur "vivant" de diffusion de l'offre en assurant une communication vivante de leur spectacle. De cette façon, les élèves du théâtre-école du Sphinx à Nantes assurent eux-mêmes la promotion de toutes les pièces en présentant des saynètes de manière itinérante dans le centre-ville. Ces « parades » inspirées du festival d'Avignon complètent l'affichage et la distribution de tracts de manière efficace puisque, selon le directeur du théâtre, une heure de parade permettrait d'attirer environ dix personnes sur le lieu du spectacle.

Des lieux de cour de cible seraient à investir pour diffuser de l'information. Par exemple, la promotion des spectacles vivants par le biais d'affiches ou de tracts dans les milieux associatifs où se pratiquent ces activités (écoles de danse, ateliers de théâtre) permet de toucher les non-consommateurs sensibles à cette forme artistique. Les initiatives de communication de la programmation de spectacles dans des guides citadins disponibles dans les lieux fréquentés (bars, restaurants, maisons de presse, boutiques de décoration ou de vêtements) vont également dans ce sens.

Enfin, des propositions " hors les murs » sont à étudier de manière à jouer sur les lieux de diffusion des œuvres; une logique de flux pourrait ainsi être envisagée de façon à 
placer l'œuvre là où se trouvent les spectateurs potentiels. C'est le cas à Nantes du «Lieu Unique » où les espaces de spectacle (dédiés à la danse, au théâtre et à la musique) sont imbriqués dans des lieux de vie (bar, restaurant, librairie, crèche, hammam). Ces derniers n'ont pas été conçus pour rester en marge des espaces réservés à la création artistique, ils s'y intègrent au contraire parfaitement. Le « Lieu Unique », aménagé dans l'ancienne usine LU, est conçu comme un ensemble mixte où la culture est au cœur de la vie. Ceci est renforcé par l'architecture du lieu conçu avec des espaces modulables (cloisons mobiles, plateaux démontables, gradins télescopiques). Le lieu peut ainsi jouer un rôle essentiel pour créer de nouvelles formes de proximité affective et faire tomber des codes perçus comme contraignants dans la relation à l'œuvre (15).

Enfin, quelles que soient les stratégies adoptées (action collective, accompagnement, auto-détermination ou communication expérientielle), les actions engagées devront concourir à faciliter l'appropriation de l'œuvre en permettant aux individus de se sentir chez eux (ce que Carù et Cova (4) nomment « la nidification »), en les guidant dans leur parcours de découverte et d'acquisition des connaissances («l'exploration»), en les aidant à attribuer un sens subjectif à l'expérience vécue (« le marquage »).

\section{Conclusion}

L'objectif de la recherche a été d'explorer les freins à la consommation des spectacles vivants pour les non-publics. En adoptant une démarche exploratoire inductive, l'enjeu fut de les identifier à travers la conception que les individus ont de l'art. Au terme de l'analyse de soixante-huit entretiens non-directifs auprès de non-consommateurs de spectacles vivants, nous avons montré que :

- deux conceptions dissonantes de l'art coexistent pour ces individus : l'une individuelle et affective associée à la musique et à la peinture, l'autre collective et cognitive associée au spectacle vivant ;

- ces conceptions renvoient aux dimensions de la valeur perçue de l'expérience de consommation puisque les représentations individuelles et affectives dévoilent une conception de l'art intrinsèque, orientée vers soi et active, alors que les représentations collectives et cognitives dévoilent une conception extrinsèque, orientée vers les autres et passive ;

- le spectacle vivant, s'inscrivant dans les représentations cognitives et collectives de l'art, induit une relation distanciée avec l'œuvre en raison de pratiques 
sociales perçues comme très normées, donc contraignantes. Ces normes, entravent l'appropriation de l'œuvre et constituent par conséquent des freins à la consommation.

L'enjeu pour les professionnels de la culture est donc de mettre en œuvre des actions auprès des non-spectateurs afin de favoriser l'appropriation cognitive et affective de cette forme d'art. Plusieurs axes ont été proposés : favoriser la création de contextes expérientiels d'une part et assurer la diffusion des œuvres sous la forme de communication expérientielle d'autre part.

Les limites de la recherche sont inhérentes à son format exploratoire. Pour confirmer les résultats et tester leur validité externe, il pourrait être intéressant de tester la contribution du contenu des représentations à la formation de la valeur perçue de l'expérience. Il serait en outre pertinent d'élargir l'investigation en cherchant à discriminer les non-consommateurs selon une typologie construite de critères d'accès à la culture (rôle actif de prescription familiale ou amicale, influence de la formation artistique...). D'autre part, une recherche est actuellement menée avec un diffuseur de spectacles vivants afin de tester les recommandations managériales présentées ici. L'analyse de ses résultats permettra d'améliorer la compréhension des mécanismes psychologiques qui tiennent les individus à distance des spectacles vivants. L'enjeu est essentiel pour les professionnels de la culture, qui doivent réinventer leurs relations avec leurs non-publics afin de conserver leur légitimité.

\section{Références}

(1) Bourgeon D., Filser M. et Pulh M. (2003), Le marketing du spectacle vivant, Revue Française de Gestion, 29, 142, 113-128.

(2) Bourgeon-Renault D., Debenedetti S., Gombault A., Petr-Le-Huérou C. (2009), Marketing de l'art et de la culture, Paris, Dunod.

(3) Carù A. et Cova B. (2003), Approche empirique de l'immersion dans l'expérience de consommation : les opérations d'appropriation, Revue Recherche et Applications en Marketing, 18, 2, 47-65.

(4) Carù A. et Cova B. (2006), Expérience de marque : comment favoriser l'immersion du consommateur ?, Revue Décisions Marketing, 41, 43-52.

(5) Coulangeon P. (2005), Sociologie des pratiques culturelles, Paris, La découverte, Collection Repères.

(6) Donnat O. (2004), Les univers culturels des Français, Sociologie et sociétés, 36, 1, 87-103.

(7) Donnat O. et Totila P. (2003), Le(s) public(s) de la culture, Paris, Presses de Science Po. 
(8) Festinger L. (1957), A Theory of Cognitive Dissonance, Stanford, California, Stanford University Press.

(9) Gallen C. (2005), Le rôle des représentations mentales dans le processus de choix, une approche pluridisciplinaire appliquée au cas des produits alimentaires, Revue Recherche et Applications en Marketing, 20, 3, 59-76.

(10) Godey B. et Lagier J. (2002), Le rôle de «l'expérience esthétique » dans la

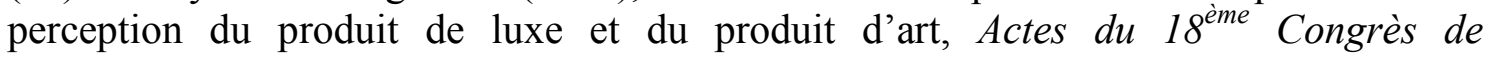
l'Association Française du Marketing, Lille, 499-518.

(11) Holbrook M.B. (1999), Consumer Value : A Framework for Analysis and Research, London \& New-York, Collection Routledge Interpretive Market Research Series.

(12) Lagier J. (2006), Le style esthétique, caractéristique individuelle de la perception et du jugement à l'égard de l'objet, Thèse de Doctorat en Sciences de Gestion, Université de Bourgogne.

(13) Lahire B. (2004), La culture des individus. Dissonances culturelles et distinction de soi, Paris, La découverte.

(14) Marteaux S. et Mencarelli R. (2004), Proposition d'enrichissement du concept de valeur dans le domaine culturel, Actes des $9^{\text {èmes }}$ Journées de Recherche en Marketing de Bourgogne, Dijon, 62-82.

(15) Mencarelli R. (2008), L'interaction lieu-objet comme conceptualisation de l'expérience vécue : test d'un modèle intégrateur, Revue Recherche et Applications en Marketing, 23, 3, 51-69.

(16) Pellemans (1999), Recherche qualitative en marketing: perspective psychoscopique, Paris, De Boeck Université. 
Annexe 1

Caractéristiques de l'échantillon

\begin{tabular}{|c|c|c|}
\hline $\begin{array}{c}\text { Tranches } \\
\text { d'âge }\end{array}$ & Professions des 34 femmes & Professions des 34 hommes \\
\hline 20-29 ans & $\begin{array}{l}\text { Etudiante en mathématiques } \\
\text { Etudiante en action commerciale } \\
\text { Orthophoniste } \\
\text { Designer scénographe } \\
\text { Assistante technique à la Sécurité } \\
\text { Sociale } \\
\text { Vendeuse en mercerie } \\
\text { Elève infirmière } \\
\text { Clerc de notaire } \\
\text { Médiatrice culturelle }\end{array}$ & $\begin{array}{l}\text { Etudiant en psychologie } \\
\text { Etudiant en sciences } \\
\text { Horticulteur } \\
\text { Animateur audiovisuel } \\
\text { Agent de développement } \\
\text { animation de proximité } \\
\text { Psychologue } \\
\text { Agent manutentionnaire }\end{array}$ \\
\hline 30-39 ans & $\begin{array}{l}\text { Assistante de direction } \\
\text { Vendeuse en électroménager } \\
\text { Aide soignante } \\
\text { Coiffeuse } \\
\text { Femme au foyer } \\
\text { Institutrice } \\
\text { Animatrice socio culturelle } \\
\text { Femme de ménage } \\
\text { Kinésithérapeute }\end{array}$ & $\begin{array}{l}\text { Brocanteur } \\
\text { Concepteur de décors sur cierges } \\
\text { Educateur sportif } \\
\text { Ingénieur en agro-alimentaire } \\
\text { Sans profession } \\
\text { Vendeur en viennoiserie } \\
\text { Chef d'entreprise } \\
\text { Chômeur } \\
\text { Chaudronnier soudeur }\end{array}$ \\
\hline$\overline{40-4}$ & $\begin{array}{l}\text { Femme au foyer } \\
\text { Correspondante de presse } \\
\text { Conseillère de clientèle en banque } \\
\text { Vendeuse indépendante à domicile } \\
\text { Maitre de conférences en } \\
\text { psychologie sociale } \\
\text { Infirmière en retraite } \\
\text { Inspectrice du trésor public } \\
\text { Consultante } \\
\text { Manipulatrice radio }\end{array}$ & $\begin{array}{l}\text { Jardinier } \\
\text { Ingénieur } \\
\text { Commercial } \\
\text { Commercial dans l'agro- } \\
\text { alimentaire } \\
\text { Viticulteur } \\
\text { Militaire de carrière } \\
\text { Plombier chauffagiste } \\
\text { Agent d'entretien et d'accueil }\end{array}$ \\
\hline $\begin{array}{l}50 \text { ans et } \\
\text { plus }\end{array}$ & $\begin{array}{l}\text { Professeur de collège } \\
\text { Médecin du travail } \\
\text { Femme au foyer } \\
\text { Hôtesse d'accueil retraitée } \\
\text { Médecin retraitée } \\
\text { Femme au foyer } \\
\text { Retraitée ancienne garde barrière }\end{array}$ & $\begin{array}{l}\text { Magistrat } \\
\text { Radiologue } \\
\text { Cafetier } \\
\text { Ingénieur } \\
\text { Responsable de fabrication dans } \\
\text { l'industrie } \\
\text { Tapissier décorateur } \\
\text { Chauffeur routier } \\
\text { Trésorier principal } \\
\text { Curé d'une paroisse } \\
\text { Imprimeur retraité }\end{array}$ \\
\hline
\end{tabular}


Annexe 2

Classification descendante hiérarchique

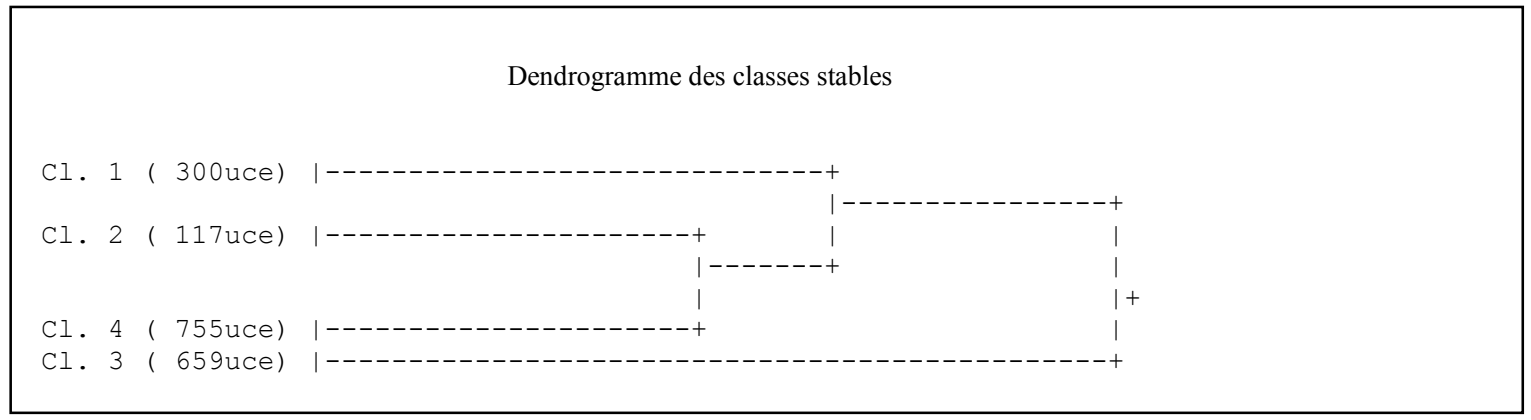

